\title{
Transmission of Emergency Medical Messages for Patient Using Control Signal of Cellular Network
}

\author{
Mohammed I Aal-Nouman ${ }^{1,2}$, Haifa Takruri-Rizk ${ }^{1}$, Martin Hope ${ }^{1}$ \\ 1 University of Salford, Manchester, UK \\ 2 Al-Nahrain University, Baghdad, Iraq
}

\begin{abstract}
- the health care system need to track and monitor the patient status and information. One of the most important requirement is that the patient can access to the service anywhere and anytime. Many systems use the internet to monitor the patient status, and in some areas especially ruler areas and motorways the internet may not available or in some cases the network might be overloaded, so the patient information cannot be delivered to the hospital or medical centre. We proposed a new method that use the spare extension of the random access channel (RACH) which is carried by physical random access channel (PRACH) to send the patient information to the medical centre. We presented the mathematical model of the channel and compare the results with another system to evaluate the results. The results shows the proposed method needs less time to transmit the patient's information.
\end{abstract}

Index Terms - emergency message, healthcare, patient monitoring, healthcare, RACH.

\section{INTRODUCTION}

The World Health Organization (WHO) has put priority for the e Health systems since 2005. eHealth is any technology that used and secure information and communication to support any field related to health, like health monitoring and surveillance, health-care services, and health education [1].

One of the most important requirement for healthcare that people can access and be provided healthcare's services anytime and anywhere. The growth of the mobile device leads to various mobile applications for healthcare services that may fulfil this requirement like Emergency Medical Services (EMS) and patient tracking and monitoring [2]. One of the major challenge is to provide healthcare services to the patients anytime [3, 4]. That means the e-healthcare systems must secure a connection and communication line between the patient and the healthcare centre. Most of the application and systems used in the health care services used the internet to send the patient monitoring information to the hospital [2, 4-8]. While there are some application used another methods to send those information to the hospital like data over voice [9].

This work is partly supported by the Ministry of Higher Education and Scientific Research of Iraq. Grant Number 3892/20-08-2013.”

Mohammed I Aal-Nouman is a PhD student in the University of Salford, Manchester UK, M5 4WT (e-mail: m.i.aal-nouman@edu.salford.ac.uk) and a Lecturer in Al-Nahrain University, Baghdad, Iraq
In many cases the patient may be in a ruler area that has no internet connection or in an area where the network is overloaded. In this case the patient information cannot be sent to the hospital or to the medical centre.

When the internet channel of the mobile network is used to send user information to the any service provider, the mobile operator get the information and then forwarded them to the service provider. To establish a connection between the user and the mobile operator network the Radio Resource Control (RRC) is used, and then the data go through another procedure and steps, in the proposed system when the RRC connection used the patient information will be sent along with the other control information to the mobile core network. The main contribution of this paper is that the patient information can be sent during RRC connection procedure using the control channels instead of the normal channels or the internet. This means that even if the network is overloaded or there is no internet connection, the information can be delivered to the hospital.

We proposed a solution to send the patient information or message to the medical service centre by using the spare extension of the random access channel (RACH) of the mobile network and carried by The Physical Random Access Channel (PRACH).

PRACH is used to carry the RACH which usually has control information. the RACH can be divided into a preamble part and a message part, as seen in Fig 1 [10]. The length of each preamble is 4096 chips' and has 256 signatures of 16 chips'.

The structure of the random-access message part radio frame can be seen in Fig 2, and the radio frame takes $10 \mathrm{~ms}$ or $20 \mathrm{~ms}$ in time depending on the number of a radio frame. Each $10 \mathrm{~ms}$ message part radio frame is split into 15 slots.

Professor Haifa Takruri-Rizk is lecturer in the University of Salford, Manchester M5 4WT (email: H.Takruri-Rizk@ salford.ac.uk) 


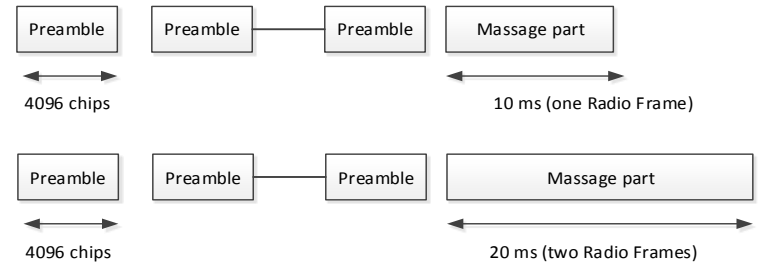

Fig 1 Structure of the RACH

each slot having a length of 2560 chips and with two parts: a control part that carries control information from the upper layer and a data part where the RACH is mapped [10].

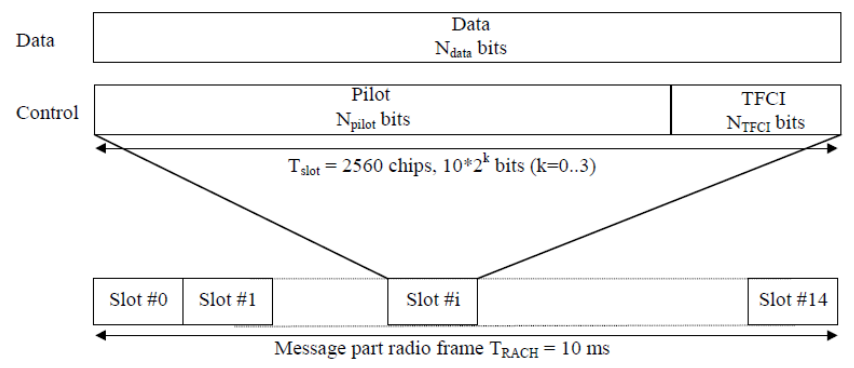

Fig 2 Frame structure for RACH

The data part consists of $10 * 2 \mathrm{k}$ bits, where $\mathrm{k}=0,1,2,3$. This is changeable according to the spreading factors, which are 256 , 128, 64 and 32 for the RACH for the message data part. The radio frame message part usually has $10 \mathrm{~ms}$ message parts, while a $20 \mathrm{~ms}$ message part consists of two $10 \mathrm{~ms}$ message part radio frames. Table 1 shows the characteristics of RACH for different slot formats [11].

TABLE 1 RACH CHARACTERISTICS

\begin{tabular}{|l|l|l|l|l|l|}
\hline $\begin{array}{l}\text { Slot } \\
\text { Format }\end{array}$ & SF & $\begin{array}{l}\text { Channel Bit } \\
\text { Rate (kbps) }\end{array}$ & $\begin{array}{l}\text { Channel } \\
\text { Symbol Rate } \\
\text { (ksps) }\end{array}$ & $\begin{array}{l}\text { Bits/ } \\
\text { Slot }\end{array}$ & $\begin{array}{l}\text { Bits/ } \\
\text { Frame }\end{array}$ \\
\hline 0 & 256 & 15 & 15 & 10 & 150 \\
\hline 1 & 128 & 30 & 30 & 20 & 300 \\
\hline 2 & 64 & 60 & 60 & 40 & 600 \\
\hline 3 & 32 & 120 & 120 & 80 & 1200 \\
\hline
\end{tabular}

The RACH channel is used to establish a connection in the Radio Resource Control (RRC) protocol, which is used in UMTS on the air interface between the UE and Node B [12]. RRC is responsible for the control signalling that includes functions like radio bearer establishment, connection establishment and connection release, broadcast of system information, reconfiguration and release, paging notification and release, connection mobility procedures [12].

The user (UE) sends an initial signal to set-up an RRC connection; this signal is RRC Connection Request message, which is carried by $\mathrm{CCCH}$ using the $\mathrm{RACH}$.

This paper is presented as follows: section II presented selected similar research work related to emergency and health services, and section III demonstrates the proposed design and solution to improve the transmission between patient and the hospital, section VI shows the mathematical model of the uplink channel, section $\mathrm{V}$ shows system validation by comparing the mathematical model with the simulation result, then section IV shows the comparison between the result of the proposed system with the result of the standard system for system evaluation, and finally the conclusion is presented in the last section IIV.

\section{SIMILAR WORKS}

The eHealth system attracts many researchers to improve its components and architecture to fulfil its requirements, one of these requirements is the reliable connection between the patient and the hospital [3, 4].

AMMA system uses the internet of the code division multiple access (CDMA) cellular network to send patient information which use an agent based mobile middleware architecture from the ambulance [2]. Also the packet channel in the thirdgeneration $(3 \mathrm{G})$ mobile network is used in the Gallego system which delivered biomedical information from the ambulance to a hospital [4]. Prakoso also used a secure system to preserve patient medical information when sending it to the doctor in far hospital for monitoring and diagnosis, the connection used in this system is the internet [8].

Another healthcare system framework is designed by Haung et al. to collect medical data from Wireless Body Area Network sensors, and send theses information to a gateway in a secure way to protect the user information and then send it to the hospital using the internet or the packet channel of the CDMA [13]. GPS and GSM network positioning technologies can be used to identify wheelchair location information, as presented in Tian et al. system, data is sent via a GSM/GPRS module, connected to a microcontroller, to the web-based management server. The server then receives the wheelchair's information and displays its location using Google Maps [6].

vNurse system is presented by Rehunathan et al, which is based on a smartphone platform that make a secure patient remote monitoring outside a clinic or hospital. It uses full Internet Protocol (IP) for the connection, and uses attached WBANs to check the patient situation while he/she is a way [14].

In some situation a quick medical responses is needed. Patients being taken to hospitals by cars could get stuck in a traffic [5]. For this reason Misbahuddin et al. presented a new scheme to transfer patient signs to the hospital using the ZigBee, if the ZigBee not available the Wi-Fi will be used and if the Wi-Fi is not present the cellular network will be used after classifying patients based on their condition [5]. Brahmi et al also proposed a similar enhanced scheme for IEEE 802.15.4 protocol to ensure fast transmission of what on road in event of car accident to the emergency service. By using this scheme the time delay will be shorter to carry important massage information [15].

There are other researcher who suggest other ways to transmit the information and not all these researchers used their system for health service. But it still can be considered as data transmission that should be taken in the consideration, Ali et al. used the multiple frequency-shift keying M-FSK modulation to transmit the data over the voice channel of the mobile network using optimised parameters in this system a new hardware is needed like a modem to let the voice channel to carry the data 
[16]. Also Ghosh et al. built a system that uses the voice channel of the mobile network to connect the central location centre with the users [17]. Peric et al. designed a system that uses the GSM network voice channel to transmit a small amount of data [18].

In the proposed method, the spare extension of the random access channel (RACH) is used to carry the patient information.

\section{PROPOSED TRANSMISSION METHOD}

When the system wants to transfer the patient information to the hospital, the information first goes to the serving mobile operator core network and the operator forward it to the hospital. In the uplink, the mobile phone or any used device sends the user information to the mobile operator through several stages. See Fig 3

The process where how the message is triggered to be sent to the hospital can be either automatically like when there is an accident, or manually that the patient send it, or regularly which is automatically sent during each intervals to monitor the patient status.

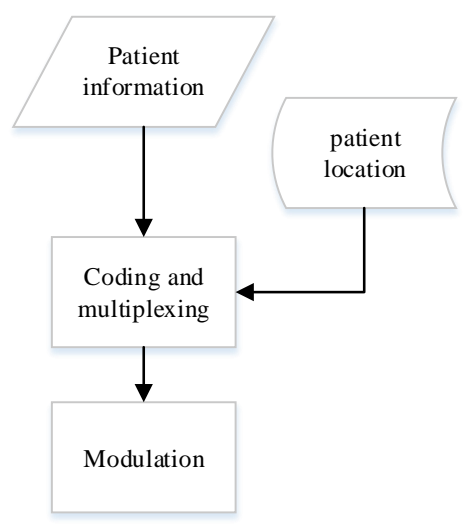

Fig 3 proposed design stages

In the beginning, the patient's mobile phone sends a message to the core network; the mobile network then locates the patient and sends this information together to the hospital.

The second step is to convert the patient message to binary and then organise this information and insert it in the spare extension in the RACH frame. The data then separates into multiple slots and mapped into the PRACH. If the data cannot fit in one slot it will take the next slot, and if all slots in one frame are filled, the data will take the second next frame, as shown inFig 4. As the RACH, which is carried by PRACH, is used to transmit control information [19] and because the RACH is the first signal sent to the operator, the proposed design uses the RACH for the uplink to carry the patient information [19-21].

Finally, those data are converted into symbols to be modulated and transmitted. The UMTS uses a root-raised cosine filter for pulse shaping and QPSK in the uplink for modulation $[21,22]$.
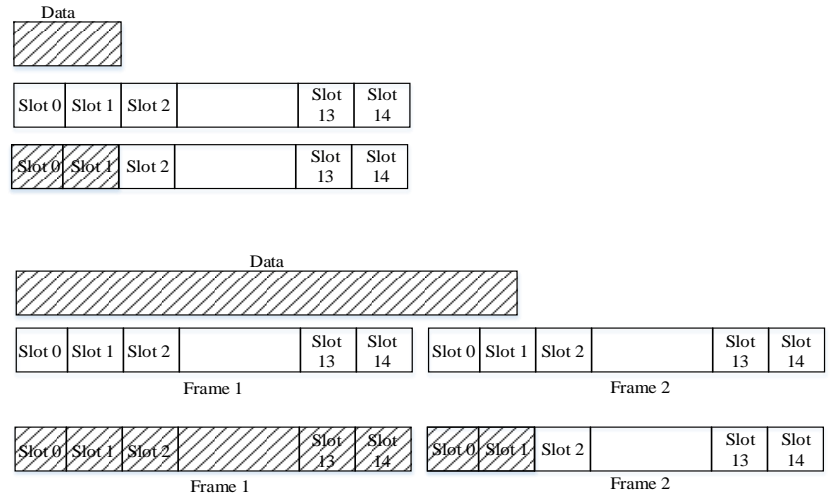

Fig 4 data mapped into slots and frame

The size of the frame is changeable depending on the spreading factors of the system and frame configuration; the NEW IEIF field in the payload can be conFigd to change the frame configuration where if bit 0 of New IEFI is set to one, the Cell Portion ID field is present. Otherwise, the field will not present. Moreover, if bit 1 of New IEFI is set to one the Ext Propagation Delay IE is present and when it sets to zero the field is not present [11]. Fig 5 shows the payload part or the $\mathrm{RACH}$.

\begin{tabular}{|c|c|c|c|c|c|c|c|}
\hline $\begin{array}{l}\text { New } \\
\text { IE FI } \\
7(E) \\
\end{array}$ & $\begin{array}{c}\text { New } \\
\text { IE FI } \\
6 \\
\end{array}$ & $\begin{array}{c}\text { New } \\
\text { IE FI } \\
5 \\
\end{array}$ & $\begin{array}{c}\text { New } \\
\text { IE FI } \\
4 \\
\end{array}$ & $\begin{array}{c}\text { New } \\
\text { IE FI } \\
3 \\
\end{array}$ & $\begin{array}{c}\text { New } \\
\text { IE FI } \\
2 \\
\end{array}$ & $\begin{array}{c}\text { New } \\
\text { IE FI } \\
1 \\
\end{array}$ & $\begin{array}{c}\text { New } \\
\text { IE FI } \\
0 \\
\end{array}$ \\
\hline Spal & e 7-6 & \multicolumn{6}{|c|}{ Cell Porting ID } \\
\hline \multicolumn{6}{|c|}{$\begin{array}{l}\text { Spare } 7-1 \text { bits for } 3.84 \text { Mcps } \\
\text { Spare } 7-2 \text { bits for } 7.68 \text { Mcps }\end{array}$} & \multicolumn{2}{|c|}{$\begin{array}{l}\text { Rx Timing } \\
\text { Deviation } \\
\text { (continuation) }\end{array}$} \\
\hline \multicolumn{6}{|c|}{ Spare 7-2 } & \multicolumn{2}{|c|}{$\begin{array}{c}\text { Ext Propa- } \\
\text { gation Delay }\end{array}$} \\
\hline \multicolumn{8}{|c|}{ Ext Propagation Delay } \\
\hline \multicolumn{6}{|c|}{ Spare $7-2$} & \multicolumn{2}{|c|}{$\mathrm{AOA}$} \\
\hline \multicolumn{8}{|c|}{$\mathrm{AOA}$} \\
\hline \multicolumn{3}{|c|}{ Spare 7-5 } & \multicolumn{5}{|c|}{$\begin{array}{c}\text { Ext Received SYNC UL Timing } \\
\text { Deviation }\end{array}$} \\
\hline \multicolumn{8}{|c|}{ Ext Received SYNC UL Timing Deviation } \\
\hline \multicolumn{8}{|c|}{ Cell Portion LCR ID } \\
\hline \multicolumn{8}{|c|}{ Spare Extension } \\
\hline \multicolumn{8}{|c|}{ Payload CRC } \\
\hline \multicolumn{8}{|c|}{ Payload CRC (cont) } \\
\hline
\end{tabular}

Fig 5 RACH payload

The frame is divided into two main sections: header and payload. At the header the FT is set to 1 if the frame carries data, or 0 if it carries control signals. The header's CRC is calculated and filled in its field after the header is filled with needed information. Fig 6 shows the RACH header.

\begin{tabular}{|c|c|c|}
\hline \multicolumn{2}{|c|}{ Header CRC } & $\mathrm{FT}$ \\
\hline \multicolumn{3}{|c|}{ CFN } \\
\hline spare & TFI & \\
\hline \multicolumn{3}{|c|}{ Rx Timing Deviation } \\
\hline Rec & g Dev & \\
\hline
\end{tabular}

Fig 6 RACH Header

The spare extension at the payload in normal and standard systems is set to zeros and sent to the receiver. It can carry up to 28 bytes of information [11]. while in the proposed design the spare extension is used to carry the patient information. 
Moreover, it depends on the size of the patient information, as the spare extension can carry up to 28 characters, whilst if the patient information is less than 28 , padding will be added to fill the spare extension.

After the data payload is filled with all necessary information and the patient information is converted into binary and put in the spare extension of the frame, the CRC calculation is resumed. The CRC is the calculation that uses polynomial division, and it is attached to the payload to check the payload error and to the header to check the header error. The polynom that is used in the header is $[11,23]$

$$
X^{7}+X^{6}+X^{2}+1
$$

And the polynom used in payload is

$$
X^{16}+X^{15}+X^{2}+1
$$

The CRC calculation starts from FT field to the last bit of the header and from the first bit to the end of the spare extension on the payload. It has two inputs and one output; the inputs are the number that we want to calculate the CRC for, and the "generator polynomial" in the form of bits is multiplied by the $\mathrm{X}^{\mathrm{n}}$.

In the payload, the size of the Transport blocks (TB) are defined and generated randomly, and the spare extension is used to send the patient information as mentioned before. The data in the spare extension can be obtained by calling a function, which gets the input from a keyboard then converts this value into binary and reshapes it to a matrix of 8 bits.

The next step is to convert the data in the frames from binary to the symbol to prepare it before it is sent to the next stages: channel coding, spreading and then modulating, where QPSK is used to modulate the signal that carries the information to the core network. The design procedure can be seen in Fig 7.

All the spreading factors for $\mathrm{RACH}$ are taken into consideration, so the spreading factors could be one of the following spreading factors $(32,64,128$ and 256). At the end of this stage, the data is transmitted from the terminal and will be managed to be forwarded to the core network by UTRAN which then forwarded it to the hospital.

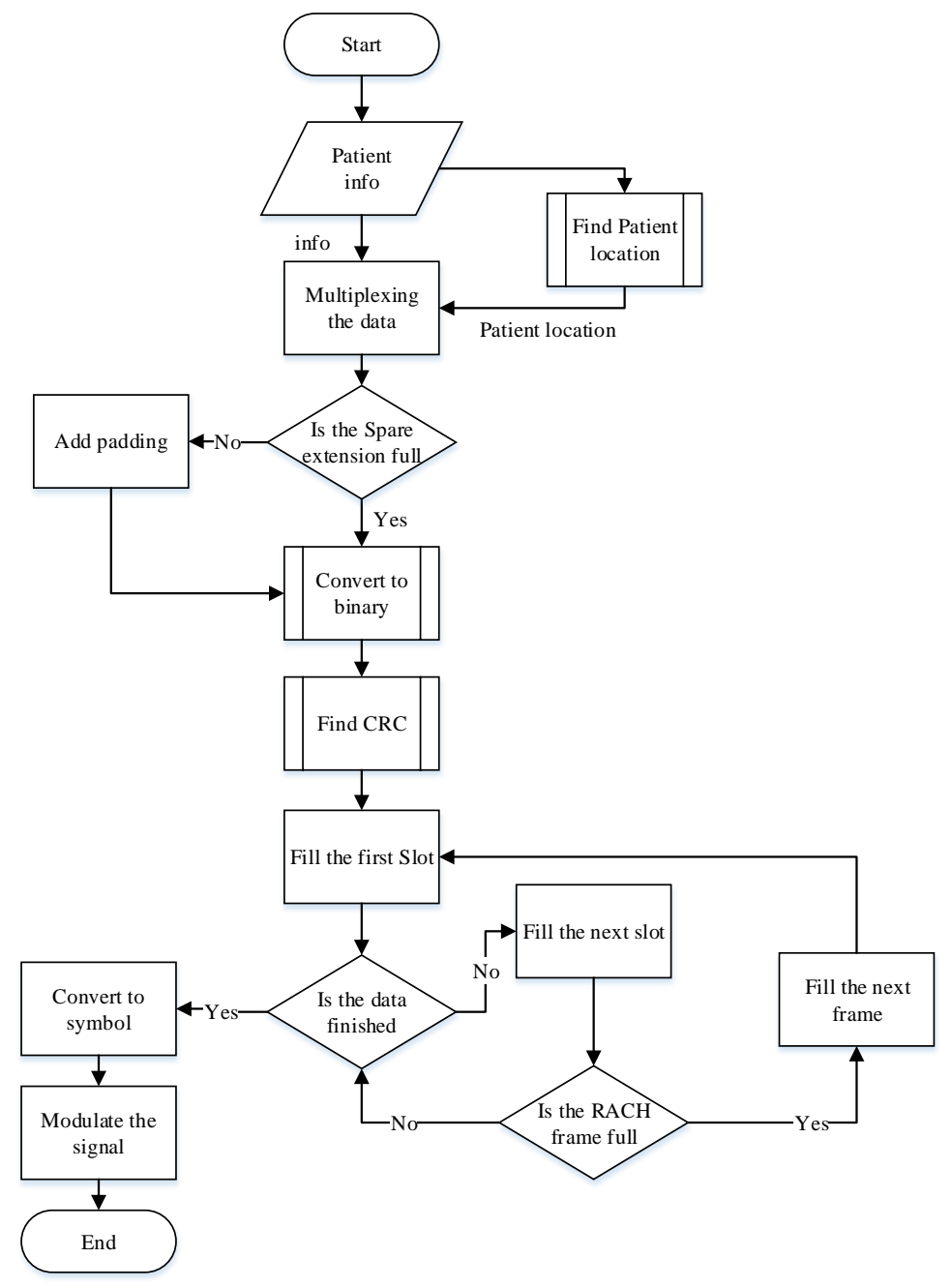

Fig 7 proposed method flowchart

\section{MATHEMATICAL ModEL}

In the uplink the data is filled in $\mathrm{RACH}$ message part and mapped into PRACH. The PRACH message part frame (A) has 15 slots (a) [10], which can be presented as

$$
A=\{a 1, a 2, \ldots, a i\}
$$

The maximum value in PRACH for $\mathrm{i}=15$, the data size of each slot can be calculated for different spreading factors sf as

$$
\text { Size }_{a}=10 * 2^{k}, \quad \forall a \in A, k \in\{1,2,3,4\}
$$

Where $\mathrm{k}$ is a variable, its value depends on the spreading factor sf. $\mathrm{k}$ can be found from the spreading factor equation as

$$
s f=\frac{256}{2^{k}} \Rightarrow k=\frac{\left(\log \frac{256}{s f}\right)}{\log 2}
$$

The slot size then can be formulated as

$$
\text { Size }_{a}=10 * 2^{\frac{\left(\log \frac{256}{5 f}\right)}{\log 2}}
$$

Because of the frame in PRACH is a group of number of slots $(m)$, where it can be up to 15 slots per frame size $\left(\right.$ Size $\left._{A}\right)$, the frame size is depends on slot size $\left(\right.$ Size $\left._{a}\right)$ and can be calculated as

$$
\text { Size }_{A}=\sum_{1}^{m} \text { Size }_{a}, \quad \text { bits where } m \leq 15
$$




$$
\begin{aligned}
& \text { Size }_{A} \sum_{1}^{m} 10 * 2^{k}, \quad \text { bits for } k \in\{0,1,2,3\}, m \leq 15 \\
& \text { Size }_{A}=\sum_{1}^{m} 10 * 2^{\frac{\left(\log \frac{256}{s f}\right)}{\log 2}}, \quad \text { bits for } s f \epsilon\{32,64,128,256\}, m \leq 15
\end{aligned}
$$

According to $3 \mathrm{GPP}$, the time needed to transmit one frame is $10 \mathrm{~ms}$ in the $\mathrm{RACH}$, so the channel bit rate $\left(C h_{\text {bitrate }}\right)$ can be calculated as:

$$
\begin{gathered}
\text { Ch }_{\text {bitrate }}=\frac{\text { Size }_{A}}{\text { Time }} \\
C h_{\text {bitrate }}=\frac{\sum_{1}^{m} 10 * 2^{\frac{\left(\log \frac{256}{\log }\right)}{\log 2}}}{10} \text { Kbps, for } s \in\{32,64,128,256\}
\end{gathered}
$$

The data that will be transmitted by PRACH is mapped from the RACH frame. To find the size of this data, the number of slots and frames needed to transmit the data must be found first. According to 3GPP the RACH frame is divided into header (Header)and payload, the payload has control fields ( $\mathrm{ctrl}$ ) which is 6 bytes length, Transport block (TB) which is 8 bits each, (CRCI) which is Cyclic Redundancy Checksum Indicator,

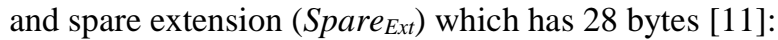

While

$$
\text { Data }_{\text {size }}=\text { Header }+ \text { Payload }
$$

$$
\begin{gathered}
\text { Payload }=c t r l+T B+C R C I_{\text {size }}+\text { Spare }_{E x t} \\
\text { Data }_{\text {size }}=\text { Header }+c t r l+T B+C R C I_{\text {size }}+\text { Spare }_{E x t}
\end{gathered}
$$

The Spare extension data size is 28 Octets. In the 3GPP standard it is sent as zeros by the transmitter and neglected by the receiver. Also, the Spare extension is trivial generally, while in the proposed design the spare extension of the RACH is used to transmit the patient information in the uplink. The calculation will be made first for the 3GPP standard and then for the proposed design. The CRCI size depends on the number of the TB as follow:

$$
C R C I_{\text {size }}=\left\lceil\frac{T B}{8}\right\rceil
$$

Substituted the new value in the equation, the payload size will be

$$
\begin{gathered}
\text { Payload }_{\text {size }}=8 *\left(6+28+T B+C R C I_{\text {size }}\right), \text {, bit } \\
\text { Payload }_{\text {size }}=8 *\left(34+T B+\left[\frac{T B}{8}\right\rceil\right), \text { bit }
\end{gathered}
$$

Because the data could take one or more frame, the number of frames needed to transmit the information without the header need to be calculated first; the data payload will be dived over the size of the frame with no header and then adding the header which is normally 4 bytes, the formula for the number of frames before adding the header will be:

$$
N_{\text {Frame }}=\left\lceil\frac{\text { Payload }_{\text {size }}}{\text { Size }_{A}-\text { Header }}\right\rceil
$$

Substitute the frame size and the payload size into the equation:

$$
N_{\text {Frame }}=\left\lceil\frac{8 *\left(6+28+T B+C R C I_{\text {size }}\right)}{\sum_{1}^{m}\left(10 * 2^{\frac{\left(\log \frac{256}{\log }\right)}{\log }}\right)-4 * 8}\right\rceil
$$

$$
N_{\text {Frame }}=\left[\frac{8\left(34+T B+\left\lceil\frac{T B}{8}\right]\right)}{\sum_{1}^{m}\left(10 * 2^{\frac{\left(\log \frac{256}{s f}\right)}{\log 2}}\right)-32}\right\rceil
$$

The data size to be transmitted can be found as the header size for one or multiple frames plus the payload size

$$
\begin{gathered}
\text { Header }_{\text {size }}=4 * 8 * N_{\text {frame }}, \text { bits } \\
\text { Data }_{\text {size }}=\text { Header }_{\text {size }}+\text { Payload }_{\text {size }} \\
\text { Data }_{\text {size }}=32 *\left[\frac{8\left(34+T B+\left\lceil\frac{T B}{8}\right\rceil\right)}{\sum_{1}^{m}\left(10 * 2 \frac{\left(\log \frac{256}{\log }\right)}{\log }\right)-32}\right]+8 *\left(34+T B+\left\lceil\frac{T B}{8}\right\rceil\right)
\end{gathered}
$$

The number of slots needed to transmit this information will be

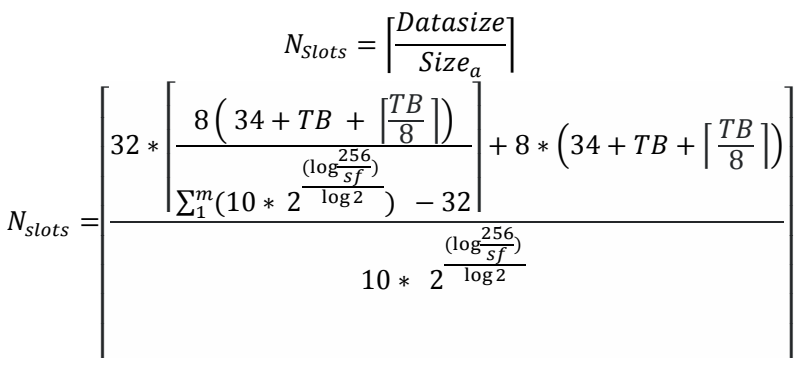

Number of frames needed to transmit the whole data after adding the header size for one or multiple frames with the payload data will be

$$
N_{\text {frame }}=\left[\frac{N_{\text {frame }}=\left\lceil\frac{N_{\text {slots }}}{15}\right\rceil}{32 *\left[\frac{8\left(34+T B+\left\lceil\frac{T B}{8}\right\rceil\right)}{\sum_{1}^{m}\left(10 * 2^{\left.\frac{\left(\log \frac{256}{s f}\right)}{\log 2}\right)}-32\right\rceil+8 *\left(34+T B+\left\lceil\frac{T B}{8}\right\rceil\right)}\right.} \underset{15 * 10 * 2^{\frac{\left(\log \frac{256}{\log }\right)}{\log }}}{15}\right.
$$

Because of the time needed to transmit a frame is 10ms [10] the time needed for one slot will be $10 \mathrm{~ms} / 15$ which is $0.667 \mathrm{~ms}$. The time needed to transmit the whole data will be:

time $=0.67 *\left[\frac{32 *\left\lceil\frac{8\left(34+T B+\left\lceil\frac{T B}{8}\right\rceil\right)}{\sum_{1}^{m}\left(10 * 2^{\frac{\left(\log \frac{256}{s f}\right)}{\log 2}}\right)-32}\right\rceil+8 *\left(34+T B+\left\lceil\frac{T B}{8}\right\rceil\right)}{10 * 2^{\frac{\left(\log \frac{256}{s f}\right)}{\log 2}}}\right]$

For the proposed method, the spare extension carries information which are the patient information, and the size of the patient information (URS) can be between 1-28 bytes, and then a padding is added to the spare extension to make it 28 byte again as it is always sent in this size, the spare extension size $(S E S)$ can found as

$$
S E S=\left(\left\lceil\frac{U R S}{28}\right\rceil * 28-U R S\right), \quad \text { byte }
$$

When using the spare extension as field to carry the patient information, the TB fields are no more needed, and any field 
related to the TB will be omitted. The payload and the frame size can be found as follow:

$$
\begin{array}{ll}
\text { Payload }=(6+\text { SES }+ \text { URS }), & \text { byte } \\
\text { Payload }=\left(6+\left\lceil\frac{U R S}{28}\right\rceil * 28\right), & \text { byte }
\end{array}
$$

To find the number of the frame needed, the payload is divided over the frame without the header

$$
\begin{aligned}
& N_{\text {Frame }}=\left\lceil\frac{\text { Payload }}{\text { Size }_{A}-\text { Header }}\right\rceil \\
& \text { Header }_{\text {size }}=4 * N_{\text {frame }}, \quad \text { byte } \\
& \text { Header }_{\text {size }}=8 * 4 *\left\lceil\frac{8 *\left(6+\left\lceil\frac{U R S}{28}\right\rceil * 28\right)}{\sum_{1}^{m}\left(10 * 2^{\left.\frac{\left(\log \frac{256}{s f}\right)}{\log 2}\right)}-32\right.}\right\rceil
\end{aligned}
$$

Now adding the header with payload to find the whole data size

$$
\begin{gathered}
\text { Data }_{\text {size }}=\text { Header }_{\text {size }}+\text { Payload }_{\text {size }} \\
\text { Data }_{\text {size }}=32 *\left[\frac{8 *\left(6+\left\lceil\frac{U R S}{28}\right\rceil * 28\right)}{\sum_{1}^{m}\left(10 * 2^{\left.\frac{\left(\log \frac{256}{s f}\right)}{\log 2}\right)}\right)-32}\right\rceil+8 *\left(6+\left\lceil\frac{U R S}{28}\right\rceil * 28\right)
\end{gathered}
$$

The number of Slots needed to transfer the data

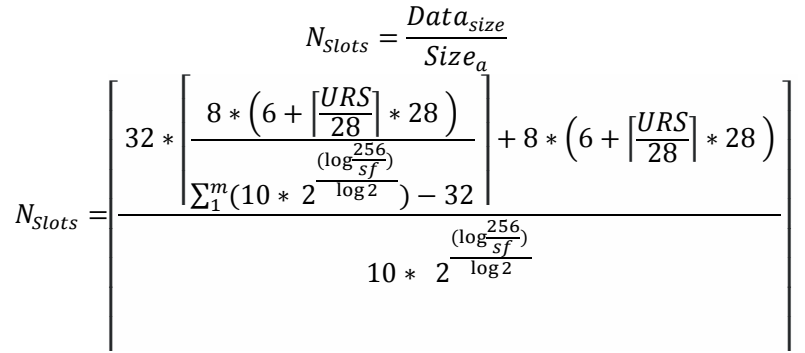

The time needed to transmit the information for the new design will be

$$
\text { Time }=0666 *\left[\frac{32 *\left\lceil\frac{8 *\left(6+\left\lceil\frac{\text { time }}{28}\right\rceil * 28\right)}{\sum_{1}^{m}\left(10 * 2^{\frac{\left(\log \frac{256}{s f}\right)}{\log 2}}\right)-32}\right\rceil+8 *\left(6+\left\lceil\frac{U R S}{28}\right\rceil * 28\right)}{10 * 2^{\frac{\left(\log \frac{256}{s f}\right)}{\log 2}}}\right]
$$

And the number of frame after adding the header size for one or multiple frames along with the payload will be

$$
\left.N_{\text {frame }}=\frac{\left[32 *\left\lceil\frac{N_{\text {frame }}=\left\lceil\frac{N_{\text {slots }}}{15}\right\rceil\left(6+\left\lceil\frac{U R S}{28}\right] * 28\right)}{\sum_{1}^{m}\left(10 * 2^{\left.\frac{\left(\log \frac{256}{s f}\right)}{\log 2}\right)}-32\right\rceil}\right\rceil 8 *\left(6+\left\lceil\frac{U R S}{28}\right\rceil * 28\right)\right.}{10 * 2^{\frac{\left(\log \frac{256}{s f}\right)}{\log 2}}}\right\rceil
$$

\section{SySTEM VALIDATION}

The simulation result obtained from the uplink channel using the RACH is compared with the mathematical model. As mentioned earlier in this chapter, the uplink can take different spreading factors, which are 32, 64, 128 and 256. Fig 8 shows the comparison between the mathematical results and the

simulation results for the spreading factor $=64$.
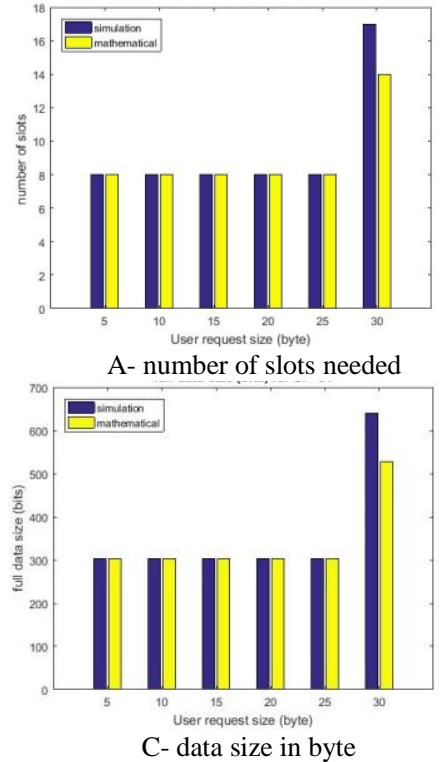

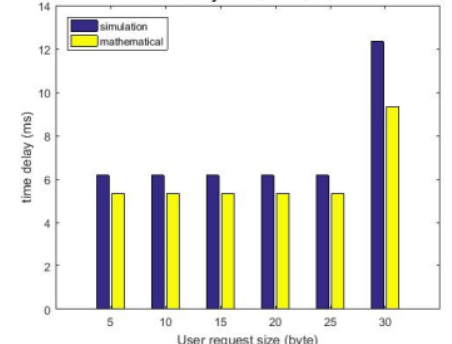

B- time needed

Fig 8 comparison between the mathematical results and simulation result for uplink with $\mathrm{SF}=64$

The Fig shows the differences between the mathematical model results and the simulation results for the uplink channel in regards to number of slots needed for PRACH to send the data (A), the time needed to send these slots (B) and the size of the data in the RACH that those slots carry $(\mathrm{C})$.

There are slight differences in the time needed to send the information (B), while the other results show a match between the mathematical and simulation results. Moreover, there are other mismatches when the patient information exceeds 28 bytes. That is because when the patient information is above 28 bytes, the system will need another set of spare extension fields, and will add it up.

Fig 9 shows the results for the spreading factor $=128$, which, it can be seen, has a similar behaviour to those in $\mathrm{sf}=64$.

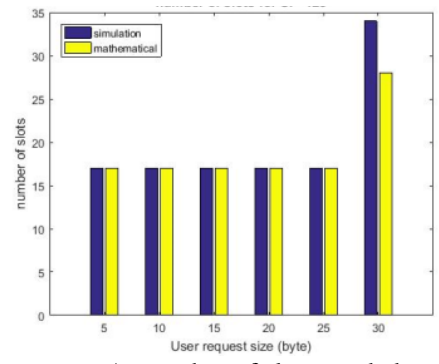

A- number of slots needed

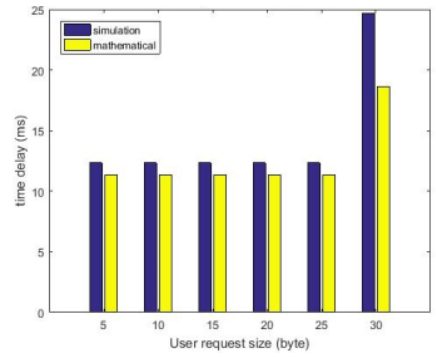

B- time needed

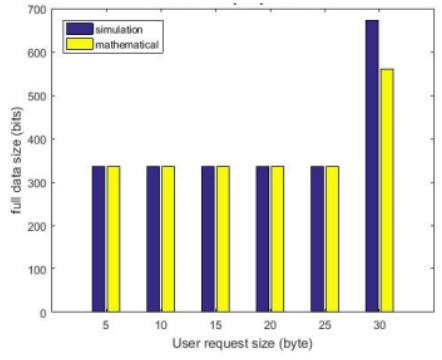




\section{C- data size in byte}

Fig 9 comparison between the mathematical results and simulation result for uplink with $\mathrm{SF}=128$

\section{System Evaluation}

The results show the evaluation results between the proposed system with the standard system, where the new system design is proposed to send the patient information using the spare extension of the RACH I uplink, while the standard sends the spare extension as zeros and uses another field in the frame structure to send the user information. Only the results for two spreading factors are shown in this section, which are $\mathrm{SF}=64$ and 128. Fig 10 illustrates a comparison between the system design and the standard with spreading factor $=64$.

The Fig shows the differences between the results from the new proposed design and the standard design as other systems for the uplink channel in respect to number of slots needed for PRACH to send the data (A), the time needed to send these slots (B) and the size of the data in the RACH that those slots carry (C).

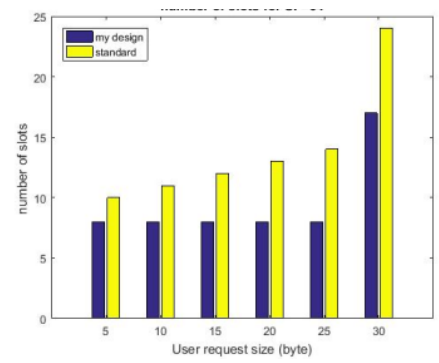

A- number of slots needed

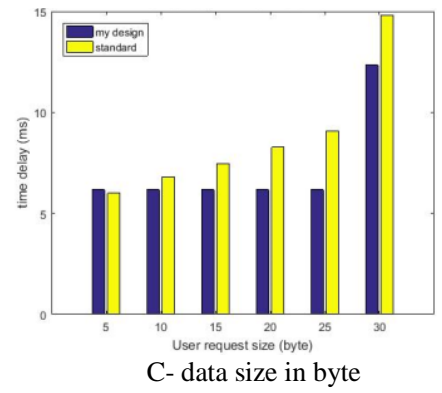

Fig 10 comparison between the simulation results of the standard and the new design for the uplink with $\mathrm{SF}=64$

It is obvious that the new design performs better than that in other systems, as the data needed to send the patient information is increasing continuously in the other system (C), while it keeps its size until it reaches 28 bytes. Consequently, the number of slots needed in PRACH to send this information is less in the proposed design (A), which leads to less time needed (B).

Fig 11 shows different values for the spreading factor, which is 128 in the uplink. And it can be seen the results are similar to those when the $\mathrm{sf}=64$.
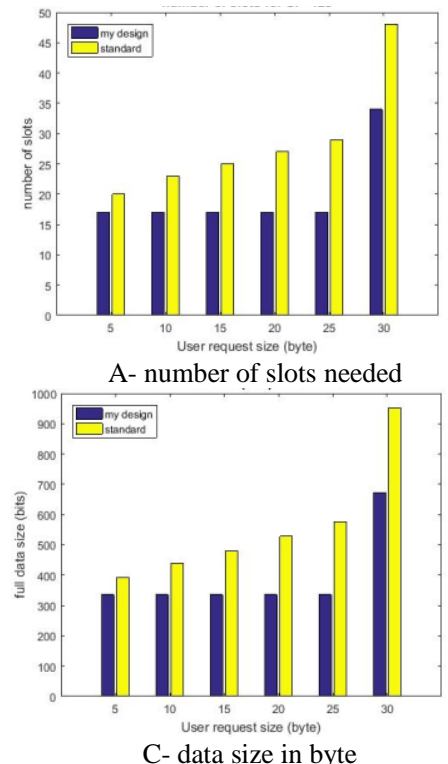

Fig 11 comparison between the simulation results of the standard and the new design for the uplink with $\mathrm{SF}=128$

\section{CONCLUSION}

This paper has presented a new method to transmit the patient information to the mobile operator to forward it to the hospital without using the internet. The main contribution of this paper is to utilize the spare extension in the RACH; instead of sending of as zeros by the sender and neglected by the receiver, a useful data will use the spare extension. That the spare extension of the RACH is used for uplink and carried by the PRACH to the core network.

In the 3GPP standards, the RACH is mainly used for RRC connection establishment procedure, and the data will sent after further steps. However, in some cases it could carries small amount of data. All the results shown for the standard uplink in this paper is only for the case where the RACH is used to carry the small amount of data because it is the fastest method, and the results from the proposed method are compared with this method.

If there are many patients sending information at the same time, the RACH uses the Slotted ALOHA methods, where when there is a collision, all the connections will be destroyed and the user is asked to retransmit with a random time [24]. So, there will be no problem for the proposed method if there is one user or multiple users.

The results show the proposed method transmit the patient information is faster than that used in the standard. Moreover, the information has delivered to the core network without using the internet.

There are many possible applications that can be used in the proposed design, like emergency services in a rural areas or on motorways where the internet coverage is not available.

\section{REFERENCES}


1. Organization, W.H., Global diffusion of eHealth: making universal health coverage achievable. 2016.

2. Arunachalan, B., J. Light, and I. Watson. Mobile Agent Based Messaging Mechanism for Emergency Medical Data Transmission Over Cellular Networks. in 2007 2nd International Conference on Communication Systems Software and Middleware. 2007.

3. Ren, Y., et al., Monitoring patients via a secure and mobile healthcare system. IEEE Wireless Communications, 2010. 17(1): p. 59-65.

4. Gallego, J.R., et al., Performance analysis of multiplexed medical data transmission for mobile emergency care over the UMTS channel. IEEE Transactions on Information Technology in Biomedicine, 2005. 9(1): p. 13-22.

5. Misbahuddin, S., et al. Client-server based transmission scheme over GSM network for MEDTOC with patient classification. in 2012 International Conference on Collaboration Technologies and Systems (CTS). 2012.

6. Tian, Z., J. Yang, and J. Zhang. Location-based Services Applied to an Electric Wheelchair Based on the GPS and GSM Networks. in Intelligent Systems and Applications, 2009. ISA 2009. International Workshop 2009.

7. Chaklader, S., et al. Black Box: An emergency rescue dispatch system for road vehicles for instant notification of road accidents and post crash analysis. in Informatics, Electronics \& Vision (ICIEV), 2014 International Conference on. 2014.

8. $\quad$ Prakoso, B.M., et al. Performance analysis of OLSR Routing for secure medical data transmission for rural areas with Delay Tolerant Network. in 2016 International Symposium on Electronics and Smart Devices (ISESD). 2016.

9. Werner, M., et al. Cellular In-Band Modem Solution for eCall Emergency Data Transmission. in VTC Spring 2009 - IEEE 69th Vehicular Technology Conference. 2009.

10. 3GPP_TS25.211, Physical channels and mapping of transport channels onto physical channels (FDD), Release 14 Version 14.0.0 2017.

11. 3GPP_TS25.435, UTRAN Iub interface user plane protocols for Common Transport Channel data streams, Release 14 Version 14.0.0. 2017.

12. 3GPP_TS25.331, Radio Resource Control (RRC) Protocol specification, Release 14 Version 14.3.0. 2017.

13. Huang, H., et al., Private and Secured Medical Data Transmission and Analysis for Wireless Sensing Healthcare System. IEEE Transactions on Industrial Informatics, 2017. 13(3): p. 1227-1237.

14. Rehunathan, D., et al. vNurse: Using virtualisation on mobile phones for remote health monitoring. in 2011 IEEE 13th International Conference on e-Health Networking, Applications and Services. 2011.

15. Brahmi, H.I., S. Djahel, and J. Murphy. Improving emergency messages transmission delay in road monitoring based WSNs. in 6th Joint IFIP Wireless and Mobile Networking Conference (WMNC). 2013.
16. Ali, B.T., G. Baudoin, and O. Venard. Data transmission over mobile voice channel based on $M$ FSK modulation. in Wireless Communications and Networking Conference (WCNC), 2013 IEEE. 2013.

17. Ghosh, R., et al., Low-complexity leakage current acquisition system for transmission line insulators employing GSM voice channel. Electronics Letters, 2015. 51(19): p. 1538-1540.

18. Peric, M., et al. An experiment with real-time data transmission over global scale mobile voice channel. in Telecommunication in Modern Satellite, Cable and Broadcasting Services (TELSIKS), 2015 12th International Conference on. 2015.

19. Springer, A. and R. Weigel, UMTS :The Physical Layer of the Universal Mobile Telecommunications System. 2002: Springer Berlin Heidelberg.

20. Aal-Nouman, M., H. Takruri-Rizk, and M. Hope. Efficient message transmission method for in-vehicle emergency service. in 2016 6th International Conference on Information Communication and Management (ICICM). 2016.

21. Aal-Nouman, M., H. Takruri-Rizk, and M. Hope. Efficient Communications for Location-Based Services Using Spare Extensions of Control Channels in Mobile Networks. in 2016 8th IFIP International Conference on New Technologies, Mobility and Security (NTMS). 2016.

22. Walke, B., R. Seidenberg, and M.P. Althoff, UMTS, The Fundamentals. 2003: WILEY.

23. 3GPP_TS25.425, UTRAN Iur interface user plane protocols for Common Transport Channel data streams, Release 14 Version 14.0.0. 2017.

24. Schiller, J.H., Mobile communications. 2003: Pearson Education. 